\title{
Universidad y búsqueda de trabajo entre los ex-alumnos que han cursado los ciclos formativos de "Servicios Socioculturales y a la Comunidad ${ }^{1}$
}

M. ${ }^{a}$ ISABEL JOCILES RUBIO

Dpto. de Antropología Social

Universidad Complutense. Madrid

\section{RESUMEN}

La Reforma Educativa, iniciada en la década de 1980, ha supuesto una gran transformación de la formación profesional reglada (FPR), tanto en lo que se refiere a los títulos que abarca como a la organización curricular. Sin embargo, para la mayoría de los alumnos de Servicios Socioculturales y a la Comunidad, en opinión de los profesores, no constituye una vía alternativa a la Universidad. En el trabajo se analizan las razones que están detrás del hecho de que los jóvenes consideren la FPR como una vía alternativa para incorporarse a los estudios universitarios.

Palabras clave: Formación Profesional, Antropología de la educación, Madrid.

\section{SUMMARY}

Education Reform in Spain, started in the 1980s, has brought about deep changes in Regulated Vocational Training (RVT) which affect the range of degrees offered as well as the curricular organization. However, teachers feel that for the majority of students enrolled for Sociocultural and Community Services, RVT is not nowadays an alternative way to university education. The author analyzes the reasons behind the students' view that RVT is no more than a round way toward a college degree.

Key words: Vocational Training, Anthropology of Education, Madrid.

\footnotetext{
${ }^{1}$ Los ciclos formativos de Animación Sociocultural (ASC), Integración Social (IS) y Educación Infantil (EI) forman parte de una de las familias profesionales (la de Servicios Socioculturales y a la Comunidad -SSC-) en que está organizada actualmente la Formación Profesional reglada, que se imparte en Institutos de Enseñanza Secundaria. Todos los ciclos formativos de esta "familia" son de grado superior, de modo que para cursarlos es preciso tener el título de bachiller (no la selectividad), haber superado algún otro ciclo formativo del mismo nivel o FPII o, en su defecto, una prueba de acceso para mayores de 20 años.
}

RDTP, LVIII, 2 (2003): 141-160 


\section{INTRODUCCIÓN}

En un artículo donde comparan los resultados de un estudio sociométrico efectuado para conocer la influencia de la etnicidad en las relaciones de amistad entre los alumnos de primaria con los obtenidos posteriormente mediante una investigación etnográfica, Denscombe et al. (1995: 146) aseguran, entre otras cosas, que la observación directa de los comportamientos en la escuela pone de manifiesto que esas relaciones responden más “a las opiniones" que el profesorado tiene sobre ellas que a lo que reflejan los tests sociométricos. A su parecer, en lugar de proceder como habitualmente se hace en la investigación educativa, es decir, desechando las opiniones de los profesores al tacharlas "bien de falta de rigor y profundidad (reservada a la investigación 'científica') o bien de estar encorsetadas por sus propias convicciones culturales (cosa que no les permite ver en sus clases la realidad..)", hay que considerarlas, si no como la descripción/explicación de los fenómenos que se estudian, sí como "algo que requiere un análisis más profundo". Si he comenzado con una referencia de esta índole al artículo de Denscombe et al., ha sido precisamente porque lo que presento a continuación tiene como punto de partida las opiniones que los profesores - en esta ocasión, de los ciclos formativos de Servicios Socioculturales y a la Comunidad (SSC) se han formado sobre las razones que mueven a los alumnos a cursarlos ${ }^{2}$. Ahora bien, el interés por tomarlas en consideración no obedece a que contradigan los resultados de alguna investigación anterior que yo misma u otros hubieran llevado a cabo, sino a considerar que merece uun análisis más profundo" el contraste que ellos establecen entre la finalidad de la formación profesional y la que, desde su punto de vista, persiguen los alumnos al matricularse en aquellos ciclos formativos.

Es que aquí, en los ciclos, excepto en el de Educación Infantil (EI), que sí salen algunos puestos de trabajo, y de Animación Sociocultural (ASC) también salen colocados algunos, pero menos, pues, es un paso a la Universidad, es un paso descaradamente. Muchos vienen directamente como una segunda oportunidad para entrar en la Universidad. (Profesor de EI y ASC del centro B)

Ten en cuenta que la procedencia de algunos alumnos que vienen a los ciclos de $\mathrm{FP}$, aun cuando las pretensiones ministeriales sean diferentes y pretendan que la

\footnotetext{
${ }^{2}$ La investigación en que se basa el artículo ha seguido una estrategia etnográfica, primándose, por tanto, la profundización en los problemas abordados sobre la posible generalización de los resultados. Se ha realizado en dos Institutos de Enseñanza Secundaria de la Comunidad de Madrid donde se imparten los ciclos formativos de Servicios Socioculturales y a la Comunidad: uno perteneciente al municipio de Madrid y el otro a su área metropolitana.
} 
formación profesional es -y es un objetivo muy loable, ¿no? - formar técnicos para que encuentren trabajo..., la procedencia de muchos es gente que no es que haya fracasado, pero que quiere ir a la Universidad y no ha pasado la selectividad. (Profesor de Integración Social - IS- y ASC del centro A)

\section{LAS OPINIONES DE LOS PROFESORES}

Durante los cursos académicos 1999-2000 y 2000-2001 realicé un conjunto de entrevistas semidirectivas a profesores de dos centros públicos de la Comunidad de Madrid que imparten su docencia en los ciclos formativos de SSC, esto es, en Animación Sociocultural, Educación Infantil e Integración Social. Estaban dirigidas sobre todo a conocer sus representaciones sobre las prácticas educativas que desarrollan, por cuanto uno de los objetivos de la investigación etnográfica en la que estaba embarcada consistía precisamente en conocer los modelos de formación que el profesorado de esa familia profesional está desarrollando, a los que quería acercarme, por un lado, observando directamente sus prácticas docentes, pero también a partir de un análisis de las representaciones que manejan sobre ellas. Aunque en dichas entrevistas no pregunté directamente por cuáles son las razones que, desde su perspectiva, llevan a los alumnos a inscribirse en los ciclos formativos, se trata de un tema que surgió de diversas maneras y en distintos momentos ${ }^{3}$, sobre todo - si bien no exclusivamente- cuando hablaban de los obstáculos con que se encuentran para alcanzar algunas de las metas de enseñanza/ aprendizaje que se proponen y las metodologías educativas y/o formas de evaluación que consideran más adecuadas para ello. En este contexto discursivo, las razones de los alumnos para inscribirse en los ciclos formativos que los profesores mencionaron con más frecuencia fueron, por una parte, el haber suspendido selectividad y/o el deseo de (o, en ciertas ocasiones, "obsesión" por) acceder a la Universidad a través de ellos y, por otra, ciertas motivaciones que les llevan a elegir un ciclo de la familia profesional en lugar de otro, hasta el punto de sentirse especialmente "frustrados" cuando no pueden cursar el que han escogido en primera opción: la atracción que sobre ellos ejercen ciertos colectivos como los niños y los marginados (en el caso de los que eligen EI e IS) o el gusto por lo lúdico, por "lo informal" (en los de ASC).

Me centraré en el primer tipo de razones (que, en unas ocasiones, se presentan en términos de precedentes - porque han suspendido la selectividad $-\mathrm{y}$, otras, en términos de finalidad —hacen esto sólo para ir a la Uni-

${ }^{3}$ Una vez surgido, sí que dediqué algunos minutos a que los entrevistados especificaran su posición al respecto. 
versidad $ఐ$ ), porque son las que apuntan de modo más directo a la cuestión de la que aquí me voy a ocupar: por qué y para qué los alumnos hacen los ciclos formativos de SSC, y no tanto por qué hacen uno u otro, si bien ambos tipos de razones se confunden en algunas argumentaciones de los profesores. Un enfoque que obedece, además, a que ellos establecen una dicotomía entre las metas de los alumnos y lo que el sistema educativo perseguiría con la FP: los primeros buscarían acceder a la Universidad mientras que el segundo intentaría - utilizando una expresión entresacada de sus palabras"formar técnicos para que encuentren trabajo". Si se tiene en cuenta que el Nuevo Programa Nacional de Formación Profesional (2000: 45), por ejemplo, estipula como objetivo general de la formación profesional inicial/reglada "ofrecer cualificaciones profesionales que garanticen la empleabilidad de sus titulados", y que lo que los profesores atribuyen a los alumnos son motivaciones para cursar los ciclos formativos, cabría pensar que la dicotomía es más aparente que real; simplemente se trataría de referencias a diferentes aspectos de la realidad: a los resultados esperados por una institución educativa o a las finalidades individuales de los beneficiarios. Una cosa y otra no son, en principio, incompatibles, pues aunque los alumnos deseen acceder a la Universidad", la FP podría estar consiguiendo sus resultados. No obstante, el problema está en que cuando los profesores señalan el contraste no apuntan a la "empleabilidad", entendida como la adquisición de las capacidades necesarias para la inserción laboral, sino a las distintas trayectorias educativo-laborales que, para el momento del egreso, estarían en el horizonte trazado por unas y otras metas, así como al impacto que éstas pudieran tener en dichas trayectorias: de cumplirse el objetivo de la FP, los alumnos deberían, por un lado, hacer los ciclos "porque quieren trabajar" $y$, por otro, si no insertarse en el mundo laboral (algo que no depende sólo de su voluntad), al menos dedicarse a buscar trabajo al terminar; y no, como sucede de hecho, que la mayoría se matricula inmediatamente en la Universidad.

Creo que hay muy poquita gente que va a la FP convencida de que quiere hacer FP. Y los que van a los ciclos... van y después se matriculan otra vez en selectividad para ver si sacan la selectividad. Y si la sacan, se van a la Universidad, y otros utilizan los ciclos como paso para la Universidad. Yo no tengo datos, pero hay una mayoría que hace eso... Vamos, yo sólo conozco un caso que aprobó sin problemas y dijo que quería hacer formación profesional porque quería trabajar. (Ex-profesora de ASC del centro A)

Hay una proporción importante que hacen esto sólo para ir a la Universidad. Te hablo de Animación y de Integración, pero quizá en Animación más, y quizá también... porque no suele ser elegido en primera opción. En IS, la mayoría de la gente lo ha elegido en primera opción, casi todos... Y el hecho de que lo hayan elegido en primera opción hace que una proporción importante —que este año, pues, puede estar en torno a 9, al veintitantos por ciento- lo ha elegido porque 
quiere trabajar en esta historia... Lo cual no significa que luego no quieran seguir estudiando, a lo mejor, en la Universidad, pero en principio su meta es hacer esto y poder trabajar en ello. (Profesor de IS y ASC del centro A)

Ha de decirse incidentalmente que esta interpretación que hacen los profesores del cumplimiento de los objetivos de la FP inicial/reglada en función de sus impactos sobre las trayectorias laborales de los alumnos no está muy alejada de la que se extrae tras analizar los modelos que diferentes expertos u organismos brindan para evaluarlos. Así, por ejemplo, Ernesto Abdala (2001: 35 y ss.), de la CINTERFOR-OIT, hace la propuesta de medir el impacto sobre los "beneficiarios" de los programas de formación de jóvenes a través de tres variables: "rol ciudadano" (esto es, participación en organizaciones civiles, sindicales, políticas, etc.), "ingresos" y "empleo". Esta última variable tiene como indicador central ala inserción laboral; ; central porque, sin él, sobrarían tanto la segunda variable (que alude a los ingresos en el puesto de trabajo) como los otros indicadores de la tercera: la satisfacción laboral y la valoración del trabajo en función de las necesidades y las expectativas de los jóvenes. El quid de la cuestión está en que -de hacer caso a los profesores-, entre buena parte de los alumnos que ellos forman, las expectativas no se sitúan en conseguir un determinado tipo de trabajo tras coronar los ciclos formativos, sino en prolongar su formación en otra institución educativa.

De este modo, ven "el deseo de ir a la Universidad" como un asunto delicado. Ante todo, porque "desvirtúa" —como se ha comentado- el objetivo de la FP. Los ciclos formativos de grado superior (al igual que antes la FPII) dan acceso a ciertas diplomaturas y/o licenciaturas universitarias, pero tal como ellos lo conciben, ese acceso tendría que ser "un medio" para que los titulados pudieran "mejorar" académica y socialmente, no un "fin" en sí mismo.

A mí me parece muy bien que los ciclos formativos tengan acceso a la Universidad, pero como un medio, no como un fin. Es decir, que verdaderamente hay alumnos que, cuando no aprueban la selectividad, vengan a los ciclos formativos..., bueno, es que esa gente está frustradísima. El que se les dé la oportunidad de ir a mí me parece positivo, porque si no, no pueden mejorar nunca académicamente, pero..., claro, cuando esto lo consideran como un paso a la Universidad sólamente... Yo tengo una preocupación con los Departamentos de Orientación, porque a los alumnos se lo explican: "Maneras de llegar a la Universidad: camino uno, tal; camino dos, tal", como si fueran caminos naturales para ir hasta allá. (Profesora de EI e IS del centro A)

Sin embargo, cuando muestran el "deseo de ir a la Universidad" como un problema, esto es, cuando expresan su preocupación por el hecho de que se haya convertido en "un fin", de que se "desvirtúe" el objetivo de la FP o de que los ciclos formativos sean contemplados "como caminos naturales para 
ir hasta allán, se inquietan menos —en contra de lo que cabría deducir de su recurso a estas locuciones- por los efectos negativos que ello pudiera tener para la sociedad en general o para el espíritu que animó la reforma de la FP, que por las consecuencias que -en su opinión- se derivan para determinados aspectos del proceso de enseñanza aprendizaje o para sujetos concretos como son los alumnos. Si la meta de éstos es ir a la Universidad, en primer lugar, llegan a los ciclos "frustrados", "bajos de autoestima", "decepcionados", puesto que no lo han logrado por la vía de la selectividad (que no sólo es el "camino uno", sino el más prestigiado), e incluso "despistados" o "perdidos" sobre aquello para lo cual los van a preparar.

\begin{abstract}
Hay algunos que sí; los que tienen más informaciones sí, se incorporan de una forma muy rápida, pero hay otros que ahora, a las alturas que estamos, que estamos casi a final de octubre, siguen intentando enterarse de dónde están. Yo no sé si es tanto la decepción de haber suspendido la prueba de selectividad, de que hacen esto para ir a la Universidad o el hecho de que están totalmente perdidos, porque se han apuntado a un ciclo que ha sido a título de repesca, pero los primeros meses yo creo que están despistados y, entonces, están que no saben realmente ni lo que hacen, pero luego en cuanto se dobla ya (el curso)..., empiezan a incorporarse y ya es cuando definitivamente sabes quiénes han aceptado que están aquí y - de alguna forma- van participando. (Profesor de ASC y ex-profesor de EI del centro B)
\end{abstract}

En segundo lugar - como también aseguran-, muchos sufren una ulterior "frustración", dado que las notas de corte que se exigen desde FP son muy altas, y no todos las consiguen para acceder a la carrera universitaria que se han propuesto. Y, en tercer lugar, algunos se "obsesionan" con la nota, hasta el punto de que no sólo realizan unos esfuerzos ingentes para conseguirla (no viviendo "relajados" el proceso de enseñanza/aprendizaje), sino que presionan a los profesores durante todo el curso para obtener buenas calificaciones, para "pedir una décima".

Imagínate, Terapia Ocupacional está en un 9 para entrar. Y luego, por ejemplo, yo no sé hasta qué punto todos los terapeutas ocupacionales trabajan en Terapia Ocupacional, porque muchas veces los alumnos también lo que buscan (yendo a la Universidad) es un nivel académico, porque es verdad que con un determinado nivel académico tienen acceso a determinadas plazas, sobre todo públicas. O sea, que a mí me parece que tanto empeño en eso desvirtúa, porque hacen unos esfuerzos... en sacar nota, cuando podrían estar aquí más relajados, pero no, en cada examen necesariamente tienen que sacar un 9, y en cada trabajo un 9 (Profesora de EI e IS del centro A)

Si el "deseo de ir a la Universidad" tiene consecuencias en el estado de ánimo (o en el grado de información) de los alumnos, indirectamente también las tiene en la docencia, pues — -según se afirma- esos alumnos po- 
nen resistencia a ciertas prácticas, metodologías educativas y formas de evaluación que los profesores consideraban convenientes para profesionalizarlos y/o que se apartan de las convencionales. Lo siguiente es lo que nos dice uno de ellos:

Es que, para poder pasar a la Universidad, sabes que hay que tener una nota de corte bastante alta, mucho más alta que desde bachillerato. ¿Eso qué hace? Eso afecta a la metodología del propio trabajo en grupo, y eso hace que puedan surgir conflictos, porque si yo trabajo en grupo y, al final, si comparto la nota, entonces, ¿qué es lo que me diferencia de los demás para poder acceder a la Universidad? Además, ocurre que hay personas que tienen tanto deseo de entrar en la Universidad que llega a ser una obsesión ya; entonces, todo lo que tenga que ver con trabajar con los compañeros, pues, no lo aceptan. Hay gente que viene a eso, a sacar un nueve, y lo que le importa es que, cuando expliques algo, eso suponga tener unos buenos apuntes y aparezca en algún libro. Y, claro, el problema en estos ciclos es que no hay libros. No hay libro de texto y, bueno, tampoco es que seamos partidarios de ellos... (Profesor de IS y ASC del centro A)

$\mathrm{Y}$ esto otro es lo que asegura una profesora:

Estás haciendo una actividad, y los chavales no quieren ver el sentido, sólo ven el sentido lúdico de esa actividad, y no quieren pararse a ver lo que hay detrás, ¿no? Es que yo pienso que ellos están todavía muy enganchados al sistema educativo, de seguir estudiando todavía, y que no tienen tomada conciencia de que van a ser profesionales dentro de dos años, y tal vez es un poco porque piensan que van a seguir estudiando. No hacen el ciclo muchas veces para ser profesionales. Ése es el problema, pero a mí me da igual... Pero, bueno, mucha gente tiene su chip en que: "iQué voy a ser profesional dentro de dos años!, ¡qué va!, ¡isi yo voy a seguir estudiando educación social!n. (Profesora de ASC, EI e IS del centro A)

El "deseo de ir a la Universidad" provoca "frustración", "desinformación", "desinterés" por aquello para lo que se va a preparar a los alumnos y obstáculos para desarrollar la metodología de los ciclos. En cambio, con las razones que -en opinión de los docentes- llevan a algunos a elegir un ciclo sobre otro ocurre todo lo contrario: incitan el interés por trabajar con ciertos colectivos (niños o marginados), una idea previa sobre en qué consiste dicho trabajo, incluso el gusto por ciertos aspectos de aquella metodología. No obstante, conciben estas motivaciones incluso como más problemáticas que aquella otra. Primero, porque, según se desprende de sus palabras, los efectos que tienen sobre la profesionalización de los alumnos son más graves y, segundo, porque - sin negar expresamente su origen social- las tienden a concebir como motivaciones psíquicas profundas, enraizadas en lo que denominan "configuración mental", "idiosincrasia personal" o "identificación con los usuarios" y que, precisamente por ello, son más difíciles de cambiar que "el 
deseo de ir a la Universidad, que es visto como inducido por factores sociales (ya sea en forma de valores interiorizados o de presión externa por parte de los padres o de la sociedad en general). Efectivamente, si la atracción que sienten los alumnos por lo lúdico o por determinados colectivos como los niños o marginados entraña, verbigracia, una aidentificación con los usuarios", esa atracción (y el interés inicial derivado de ella) más que un elemento a favor, constituye una traba para la profesionalización, puesto que -como siguen narrando- impide o dificulta su identificación con los profesionales que trabajan con esos colectivos que, para serlo, deben ser adultos, normalizados o formales. Por otra parte, esas "identificaciones" suelen ir acompañadas de un preconcepto erróneo sobre aquello en que consisten las intervenciones sociales y/o educativas: el trabajo con niños lo presuponen como una labor de cuidado/besuqueo, el trabajo con excluidos como ayuda a los demás" y el trabajo en ASC como "puro activismo", como un hacer por hacer. Dada esta visión distorsionada de la naturaleza de las respectivas profesiones que tales motivaciones e ideas previas inducen, estiman preciso que se produzca en los alumnos un cambio radical de las mismas; es decir, un cambio cualitativo por sustitución. Así, por ejemplo, en lo que se refiere a su imagen de las intervenciones, sostienen que se tienen que trocar en "educar niños", "trabajar profesionalmente con los demás" o "un hacer reflexivo".

En el caso del "deseo (aun obsesivo) de ir a la Universidad", por el contrario, lo que parecen propugnar es que los alumnos lo complementen con otros motivos, lo que podríamos denominar un cambio cuantitativo por adición. Y digo que lo parecen propugnar porque en ningún momento les he oído expresar que sea eso lo que defienden, sino que se trata de una inferencia que cabe extraer tanto de sus discursos como de la observación de algunos de sus comportamientos con los alumnos. De este modo, aunque digan - como más atrás- que no están de acuerdo en que "el ir a la Universidad" sea un fin, lo que más enfatizan, lo que declaran que "es una pena" - como podrá verse en una cita más adelante- es que "sea el único objetivo", que no vaya acompañado de otros.

Por otro lado, he tenido la oportunidad de observar varias situaciones en que, por ejemplo, ante la insistencia de los alumnos en seguir practicando ciertos juegos o ante su resistencia a ponerse a reflexionar sobre la actividad que habían realizado o iban a realizar, los profesores les han exhortado (en unos casos, con bastante dosis de enfado, en otros, de sorna) a que dejaran de comportarse como niños o como adolescentes ("estudiantillos de BUP", "monitores de tiempo libre"); mientras tanto, no he presenciado exhortaciones expresamente recriminatorias ante alguna manifestación de su adeseo de ir a la Universidad", sino intentos de que lo compatibilicen con la adquisición de capacidades profesionalizadoras. 
Traeré a colación, a título ilustrativo, el caso de un alumno de ASC que, a mediados del primer trimestre, se quejó ante una profesora por el hecho que ésta hubiera formado equipos de trabajo mediante métodos aleatorios, pues - según le confesó- los compañeros que le habían tocado en suerte no eran de "los más competentes", la actividad que debían llevar a cabo era evaluable y él se estaba jugando la nota que le permitiría hacer Educación Social. La profesora le aseguró que le parecía respetable su empeño en obtener una nota alta, pero que un animador sociocultural, en el ejercicio de su profesión, tiene que formar parte de diversos equipos, a cuyos componentes habitualmente no escoge, por lo cual una de las capacidades que debe adquirir consiste en integrarse en ellos y hacerlos funcionar; la nota que obtuviera, del mismo modo que el éxito de las intervenciones socioculturales, dependería en buena medida de ello. Poco más tarde, fue otra integrante del equipo quien acudió al despacho a mostrarle su protesta, como consecuencia de que ese mismo alumno se hubiera hecho cargo por completo del trabajo, dejando a los demás sólo tareas marginales (como pasarlo a ordenador); según decía, el resto de compañeros había aceptado el reparto, pero ella no y no terminaba de "entenderse" con él. La profesora convocó entonces una reunión con todos los equipos, y su alocución se centró esta vez en trasmitirles que las actividades colectivas había que realizarlas en grupo, que los resultados de éstas suelen ser mejores y que si, por cualquier razón, obtenían una nota inferior a la que aspiraban, siempre podrían compensarla con los trabajos individuales que también tendrían ocasión de acometer a la largo del curso. Si he descrito de una forma relativamente extensa este caso es porque en él se aprecia no sólo el intento de que los alumnos complementen "el deseo de ir a la Universidad" con otros objetivos, hasta el punto de que se les plantea que el medio para lograr el primero pasa por que se cumplan esos otros, sino también porque deja ver que se establecen canales (como la diversificación de los instrumentos de evaluación) dirigidos expresa —aunque no sólamente- a que los alumnos más "obsesivos" se sientan más seguros de alcanzar su meta.

¿A qué puede ser debida esta diferencia de perspectiva/actitud ante los dos tipos de motivaciones? Sin pretender agotar la pregunta ni darle una respuesta definitiva, desde mi punto de vista, se debe a tres factores principales. Uno de ellos consiste en que detrás del "deseo de ir a la Universidad", al contrario de lo que -como se ha indicado- acaece con el otro tipo, los profesores no vislumbran la existencia de ninguna visión errónea (si acaso, la ausencia de cualquier visión) sobre la naturaleza de las intervenciones sociales y educativas que potencialmente perjudique a los usuarios y que, por tanto, se interponga en su empeño de formar al abuen profesional"; objetivo que, como he tratado de mostrar en otro lugar (Jociles 2001), sustituye al de 
la "empleabilidad" en la práctica docente de los ciclos de SSC de estos dos centros. Sin embargo, resulta sorprendente que, tanto en un caso como en el otro, emprendan muy pocas actuaciones específicas orientadas a cambiar tales motivaciones o sus efectos en los procesos de enseñanza/aprendizaje. Salvo exhortaciones (individuales o colectivas) como las que he descrito más atrás o, para el caso del "deseo de ir a la Universidad", como la diversificación de los instrumentos de evaluación, sólo conozco una acción que busca explícitamente el cambio: en el ciclo de ASC del centro B, para hacer frente a la "desinformación" inicial de los alumnos, han remodelado el currículo de tres de sus módulos de tal modo que, durante el primer trimestre, los estudiantes se dedican con exclusividad a saber qué es la ASC. Es más, se diría que los profesores confían enteramente en la metodología de enseñanza/ aprendizaje que desarrollan ya no sólo para que los estudiantes adquieran las capacidades profesionales correspondientes, sino también para que, al mismo tiempo, transformen sus motivaciones, bien sustituyéndolas bien complementándolas; una metodología (caracterizada, a grandes rasgos, por una relación profesor-alumno diferente a la que han vivido en bachillerato, la obligación -no la posibilidad- del alumnado de tener un alto grado de participación e iniciativa, la realización desde un principio de actividades de intervención social, cultural o educativa, sea mediante ejercicios de simulación, sea en contextos laborales, el contacto directo con el campo profesional a través de visitas concertadas, etc.) que, según los profesores, tiene la virtud de «engancharlos" enseguida:

-Rebotados, frustrados, es decir, gente que no ha podido hacer Turismo o no ha podido hacer Psicología, pues, de ésos muchos, muchos.

—EEso se refleja en la dinámica de clase?

-Es que normalmente se enganchan al ciclo. Se enganchan bastante rápido..

- ¿Pero el equipo docente hace algo al respecto?

-Yo creo que conscientemente no lo hacemos. Lo que pasa es que, claro, tú estás acostumbrado a estudiar en un instituto,... y de repente entras en un lugar donde, de entrada, te dicen que tienes que realizar una actividad para no sé cuándo, que tienes que montar el PIJ (punto de información juvenil), que tienes que tal, que tienes unas clases en las que debes participar y tal, y yo creo que la gente se engancha enseguida. (Profesora de ASC y EI del centro A)

Yo creo que más de uno, sobre todo de Animación, sí tiene la sensación en algunos momentos de que ha llegado aquí por lo que ha llegado: porque no han aprobado selectividad y, después, porque no les han dado el ciclo que querían, y están aquí porque no les queda más remedio y sólo como puente para la Universidad... Pero, en conjunto, yo creo que sufren una transformación y, sobre todo, en el primer trimestre. En el segundo trimestre ya son otros. Y a mí me parece que también es porque la forma que nosotros tenemos de dinamizar la clase es distinta: les obligamos a hablar en público, a enrojecer delante de los compañeros y, cuan- 
do la han hecho en clase, delante del centro... De hecho, en todos los ciclos están obligados a ser participativos, a estar constantemente en busca de sus propias respuestas y, bueno, también está el hecho de que vaya formándose cierto grado de familiaridad entre ellos y sus propios profesores. (Profesor de ASC y ex-profesor de EI del centro B)

Siguiendo con el tema de cuáles son los factores que están en la base de la distinta perspectiva/actitud que adoptan los profesores ante el cambio de las motivaciones, otro de ellos consiste en que el "deseo de ir a la Universidad" — no así el resto- es contemplado bajo el prisma de la legitimidad, como un derecho (a "tener mayor prestigio social", "un sueldo mejor" $\mathrm{O}$ a que "se le reconozca una mayor cualificación profesional") que todo el mundo tiene en cuanto ciudadano, por tanto, como un "deseo legítimo":

Es un deseo legítimo, porque, bueno, todo el mundo pretende tener mayor prestigio social, ¿no?, o que se le reconozca una mayor cualificación profesional, ¿no?, y eso se traduce en tener, pues, un sueldo mejor. Es legítimo, ¿no? A mí lo que me parece que es una pena es que sea el único objetivo el ir a la Universidad. (Profesor de IS y ASC del centro A)

Con todo, estimo que esa aceptación/condescendencia se debe a un ajuste de sus actitudes (y, en general, de su modelo de formación profesional ${ }^{4}$ ) a las representaciones que manejan del funcionamiento y la estructura del mercado laboral. Dicho de otro modo, la cuestión no estriba tan sólo en que les parezca legítimo que los alumnos aspiren a los salarios, el estatus y el prestigio social que conceden las titulaciones y cualificaciones universitarias, sino que - expresado sucintamente- reconocen que, en el mercado de "lo social, estas titulaciones les abren mayores oportunidades para ocupar, incluso, puestos de trabajo en los que, en principio, sería exigible tan sólo un nivel medio, esto es, las capacidades profesionales en las que ellos los forman. Dando un paso más, diría que esa aceptación/condescendencia constituye otro aspecto de la "pertinencia cultural" de su práctica docente, esto es, de su adaptación no sólo a sus propias representaciones, sino a las que se han forjado o se van forjando asimismo los alumnos, en quienes se va agudizando a lo largo del curso la percepción previa (más o menos difusa según su grado de experiencia en ese mercado) de que, aun para encontrar trabajos de "técnico", tienen mayores posibilidades con un título universitario; agudización que, con todo, no refuerza su tendencia a proyectar hacia un futuro lejano (habitualmente la terminación de la carrera universitaria) el momento de su incorporación al mundo del trabajo. De hecho, esta última

\footnotetext{
${ }^{4}$ Descrito en Jociles 2001.
} 
cuestión es la tesis que aquí $i^{5}$ voy a defender apoyándome en un material empírico procedente de haber realizado el seguimiento de una promoción de alumnos tras haber terminado los ciclos, diez entrevistas semidirectivas a ex-alumnos y la observación directa en los dos centros donde realizaron sus estudios (el A y el B).

\section{LOS ALUMNOS "DESPUÉS" DE LOS CICLOS FORMATIVOS}

El instrumentalizar los ciclos formativos para "acceder a la Universidad" no significa que los alumnos no estén dispuestos (si no cuando comienzan el curso, sí al finalizarlo) a posponer los estudios universitarios para desempeñar un trabajo que se ajuste a sus expectativas: en unos casos, interrumpiendo la carrera recién iniciada para emprender otra compatible con el trabajo $y$, en otros, priorizando éste de modo que sólo se implican en aquellas asignaturas que su tiempo les posibilita. De hecho, entre los ex-alumnos entrevistados encontramos varios ejemplos de ello, de los cuales voy a exponer uno:

Yo había hecho el bachillerato de ciencias de la salud y mi intención era hacer enfermería. Hice selectividad, pero suspendí selectividad y, cuando suspendí selectividad, decidí hacer el módulo... Yo lo hice en función de luego entrar en la Universidad, ¿no?, de sacar una nota, la máxima nota que pudiese para luego ir a la Universidad... Lo que pasa que, al final no hice enfermería, y me puse a hacer magisterio de primaria. Bueno, acabé el módulo y estuve 8 meses cuidando a una niña hasta que entré a hacer la carrera, porque, claro, no era como ahora, que acaban las Prácticas en junio, nosotros acabábamos las Prácticas en diciembre. Hice primero de primaria; lo terminé bien, y justo me llamaron para hacer una suplencia en una escuela (infantil) privada, y la hice. Luego me dijeron que si quería quedarme, que tenían una plaza vacante, y me pasé dos años trabajando por las mañanas cuatro horas. Por eso me cambié de carrera, porque en la Facultad que yo estaba no había clases por las tardes; o sea, yo iba por las mañanas a primaria, y o cogía el trabajo o cogía la carrera, y decidí cambiarme de carrera, porque me hacía ilusión trabajar. (EI, promoción 1995-96, centro A)

La cuestión está en que ninguno de ellos deja de matricularse en la Universidad en el año académico siguiente a aquel en que termina el ciclo

\footnotetext{
${ }^{5}$ La tesis de la "pertinencia cultural" de la práctica docente, en el sentido - señalado hace un momento- de su adecuación a las representaciones que los alumnos se han forjado y se van forjando sobre el funcionamiento del mercado laboral de "lo social", la abordo en otro artículo ("Familia profesional de 'Servicios Socioculturales y a la Comunidad': ¿es la FP reglada una alternativa a la Universidad o un camino alternativo hacia ella") que será próximamente publicado en Revista de Educación del INCE (aceptado en julio de 2003).
} 
formativo, sino es por problemas académico-burocráticos (p.e., que a los que han trabajado previamente en la profesión no les llegue el certificado de convalidación de las Prácticas con tiempo suficiente para poner hacerlo) o relacionados con el trabajo que ya desempeñaban cuando cursaban el ciclo formativo. Ello es así en el caso de todos los ex-alumnos entrevistados cuya motivación para matricularse en uno de los ciclos de SSC (8 de 10) fue "acceder a la Universidad ; y viene apoyado por el seguimiento que he realizado de los alumnos de la promoción 1996-98 que hicieron, en ese centro, los ciclos de EI y ASC ${ }^{6}$. Pues, bien, entre los que responden al mismo perfil (36 de 58), sólo dos dejaron para más adelante su entrada en la Universidad, uno porque fue contratado en el lugar donde hizo las Prácticas, y el otro, que trabajaba como interino en una institución pública, porque se le presentó la ocasión de mejorar su precaria situación laboral preparando unas oposiciones. Lo más frecuente, por tanto, es que la primera oportunidad de un trabajo que les satisfaga se les presente cuando ya han comenzado los estudios universitarios; lo que explica que tres de los que he entrevistado ( 3 de 8) los estén compatibilizando con empleos, relativamente estables, como educadores infantiles o animadores socioculturales. Los demás (y los anteriores en sus primeros tiempos de Universidad), han trabajado esporádicamente tanto en el campo de la intervención social y/o educativa como en otros alejados del mismo, desempeñando lo que despectivamente llaman "trabajillos", por lo que han otorgado primacía a sus itinerarios educativos. El caso es que no se arriesgan a "quedarse en casa" buscando trabajo, por un lado, porque no están seguros de conseguirlo y, por otro, porque estarían perdiendo, a la vez, tiempo para ganar credenciales que les sirvan para lograr uno a medida de sus expectativas. Tanto es así que los que no alcanzan la nota exigida para los de FP en las Universidades públicas, terminan matriculándose en una privada y/o en una carrera universitaria distinta a la que les interesa, incluso aquéllos que, habiéndolo podido hacer con la nota de selectividad, habían desechado esa eventualidad al acabar bachillerato. Veámoslo en dos de ellos:

Yo aprobé, pero quería hacer enfermería, y la nota de selectividad no me daba para enfermería, me daba para trabajo social, pero yo quería hacer enfermería. Pedían un 6 y pico, y yo saqué un 5 y pico de media de selectividad; entonces, con un módulo podía acceder a hacer enfermería. Yo hice el módulo, pero tampoco pude hacer enfermería, porque pedían un 8 y pico con el módulo, y yo saqué un siete y medio. Ante la opción de que me daba para trabajo social de las dos formas, pues, hice trabajo social. (EI, promoción 1994-96, centro A)

Yo quería acceder a la carrera de educación social, que era también una carrera muy nueva, que acababa de salir, que - bueno- me apetecía hacerlo. Entonces,

\footnotetext{
${ }^{6}$ El ciclo de IS no se ha implantado en el centro B hasta 2000-2001.
} 
después de selectividad, no me dio la nota... Y entonces me enteré del módulo este, del módulo de FP... Yo no sabía muy bien cómo iba a ser, ni los contenidos ni eso, pero sí que entré en el módulo. Y luego, pues, también a partir de ahí, con la idea de sacar nota para entrar en educación social, que tampoco me dio la nota... Y, entonces, pues, después de las Prácticas, estuve pensándomelo, a ver cómo lo hacía y, bueno, sociología me llamaba la atención. No sabía si tenía muchas garantías o no, pero, bueno, accedí por la nota de selectividad, que ya tenía. (AS, promoción 1994-96, centro A)

En otros casos, sin embargo, comienzan una carrera distinta a la que habían planeado en un principio porque la profesión para la que se han preparado llega a atraerles lo bastante para que, aun alcanzando la nota precisa, truequen la carrera que tenían in mente por otra que estiman más cercana al ciclo formativo que han cursado:

Me presenté a selectividad, yo sí la aprobé, pero la aprobé en septiembre; entonces, yo ya no podía optar a la pública. Ya no había plazas y tal. Entonces,... en las aulas esas que te explican lo de los ciclos y todo, entonces, yo fui directamente a buscar los modulitos que había y tal... Y, claro,... me cogí el panfletillo, vi las asignaturas y tal, y dije: "Bueno, pues, ¡vale! Esto es lo mío". Entonces, me presenté a la selectividad en septiembre..., yo ya había hecho la matrícula para el módulo antes, porque yo ya sabía que no tendría plaza, y me cogieron en el módulo e hice el módulo. Pero aquí viene lo curioso. Toda mi vida deseando ser trabajadora social, y cuando terminé el módulo, decidí hacer educación social, no trabajo social..., por la diferencia que hay entre el trabajador social y el educador social, que yo vi que lo que verdaderamente me gustaba era trabajar con la gente y estar ahí. Entonces, estuve en un centro de día con inmigrantes (haciendo las Prácticas), que era niños pequeñitos; y yo, en mi enajenación mental (ríe), dije: "Pero isi a mí lo que me gustan son los niños pequeños! E hice magisterio, o sea, me metí a magisterio de EI. Estuve todo el año pasado haciendo primero de magisterio de EI, que fue el año más deprimente de mi vida, porque yo en el módulo todo lo que estudié para IS es educación no-formal.., y fue meterme en magisterio y, claro, es educación formal... Entonces, pues, nada, terminé el primer año y, como aquí (escuela de educación social) tengo amigos que hicieron IS conmigo, pues, dije: "Nada, chicos, me voy con vosotros para allá.. (IS, promoción 1997-98, centro A)

Los ciclos de SSC producen, así, una cierta vocacionalización entre quienes no la tenían previamente y/o no sabían ni siquiera en qué consistían las profesiones para las cuales forman. El fenómeno de la vocacionalización no es, desde luego, exclusivo de esta familia profesional, como tampoco de la FP; si lo traigo a colación es, porque independientemente de su carácter exclusivo o común, tiene efectos reseñables sobre las trayectorias educativolaborales de los egresados, tanto en lo que se refiere -como se ha indicado- a las carreras universitarias que escogen finalmente como, que es lo que me interesa resaltar ahora, sobre sus planes de inserción laboral. El sentirse atraídos por esas profesiones y preparados para ejercerlas constituye uno 
de los elementos (amén, sin duda, del crecimiento etario y la posesión de un título con valor profesionalizador) que contribuyen a que los alumnos que, cuando se matricularon en FP, no tenían el propósito de buscar trabajo ni tampoco se "preocupaban" por él, terminen buscándolo, tal como asegura, entre otros, la siguiente ex-alumna:

Yo, cuando hacía el módulo, es que nunca me he preocupado por eso, porque cuando íbamos a las salidas, los profesores a lo mejor preguntaban: "Bueno, ¿y qué posibilidades tienen estos chavales de trabajar aquî?...., pero yo también me veía muy joven, en el sentido de que no me urgía. No te preocupabas por el trabajo, porque en aquel momento pensabas que ibas a seguir estudiando. Cuando terminé las Prácticas, ya me preocupé, y mandé currículums y esas cosas, pero en aquel momento yo tampoco me lo preguntaba. (IS, promoción 1998-2000, centro A)

A pesar de la vocacionalización que -como repiten todos los protagonistas, incluidos los profesores- provocan los ciclos formativos, todo parece darle la razón, sin embargo, a un ex-alumno que me decía irónicamente durante la entrevista que lo importante es ser universitario, aunque sea en "filosofía aramea". Si esto se aprecia en la rápida matriculación en la Universidad, y en que acaben cursando carreras que antes habían incluso rechazado (y/o no pueden "vincular" después con el campo profesional hacia el cual se han re-direccionado), también es posible detectarlo si nos detenemos -aun brevemente- a considerar ciertos comportamientos ligados a un asunto ya apuntado: la combinación de trabajo y estudios universitarios. De hecho, unir ambas cosas se convierte en el "ideal" para los ex-alumnos entrevistados:

Al terminar el módulo, pues, mandé currículums y tal, pero no sale trabajo como integradora. A mí me encantaría trabajar y seguir estudiando... Bueno, yo doy clases de apoyo escolar, lo que pasa es que como ahí a mí me pagan muy poquito y es muy poquito tiempo, pero me gusta un montón, y si tuviera un trabajo más decente, más horas, más dinero, pues, me gustaría más. No lo veo incompatible. Vamos, yo creo que sería lo ideal. (IS, promoción 1998-00, centro A)

Un "ideal" que lo es no sólo por cuestiones económicas (con todo, fundamentales) y por constituir una forma de adquisición de experiencia laboral, sino también, porque de no proceder a esa combinación, al acabar la carrera, podrían encontrarse de nuevo en esa situación que tanto temen: "quedarse en casa". Ahora bien, se trata de una compatibilización en que los estudios universitarios son relegados a un segundo lugar —según la expresión utilizada por una informante- únicamente cuando el trabajo reúne unas condiciones mínimas en cuanto al salario, el ámbito en que se desarrolla (el de la intervención social, cultural o educativa), las competencias profesionales que otorga y/o la duración que garantiza y/o virtualmente promete. Te- 
niendo en cuenta, además, que con dejarlos en segundo lugar no se refieren a abandonarlos, sino más bien a tomárselos "mucho más relajadamente", esto es, a lo que en páginas anteriores identificábamos como trocar una carrera universitaria por otra o a cursar menos asignaturas de las correspondientes a un año académico.

Aseguran, por ejemplo, Martín y Velarde (1996) que casi la mitad de los jóvenes estudiados por ellos están dispuestos a interrumpir sus estudios en el supuesto de que consiguieran un buen empleo. Es muy posible que una proporción parecida de alumnos y ex-alumnos de esta familia profesional, enfrentados a una pregunta de este tipo, pudiera manifestarse en el mismo sentido; una pregunta que, en el caso que nos ocupa, no estaba incluida en las entrevistas, de modo que las declaraciones al respecto son más o menos espontáneas, esto es, hechas dentro de otros marcos discursivos, estimuladas por otras preguntas. Sin embargo, considero que ello sería así en tanto en cuanto las respuestas se situaran en el terreno de la posibilidad, que es aquél que crea la situación de encuesta o de entrevista cuando se interroga sobre supuestos, pero se presenta de distinta manera cuando tienen que tomar la decisión, cuando en lugar de moverse en el terreno de lo hipotético, lo hacen en el de los hechos consumados. Cuando se analizan sus trayectorias educativo-laborales, cuando se conoce - por tanto- cuál es la que han tomado ${ }^{7}$, se aprecia que, encarados realmente a la disyuntiva, ya tienen en cuenta aspectos que no suelen contemplar cuando se les ubica en el espacio de lo meramente posible: que, por muy bueno que sea el trabajo que se les ha ofertado, por mucho que responda a sus expectativas actuales, no es aquel al que aspiran para el futuro; por lo que no abandonan sus estudios universitarios, a no ser, desde luego, que tengan ya cargas familiares (que es el caso de los dos estudiantes de la promoción 1996-98 que he mentado anteriormente: ambos varones casados, uno de ellos padre de familia, y bastante mayores - de 26 a 28 años- que el resto de sus compañeros) o que después se les presenten tales cargas o una situación socioeconómica difícil.

El trabajo que anhelan para el futuro, para cuando ya piensan en uformar una familia" o en asentar su plena autonomía, tiene prácticamente las mismas características señaladas más atrás, salvo en lo que atañe, sobre todo, a la estabilidad. La que valoran en los empleos actuales (tal vez porque es la única a la que pueden aspirar) es aquella que se da en situaciones laborales en que, aun teniendo contratos temporales, existe la oportunidad de que se

7 De la promoción de 1996-98 he recogido información sistemática sobre sus conductas educativo-laborales únicamente en lo que se refiere al siguiente año después de haber acabado el ciclo formativo. En cambio, he reconstruido las trayectorias educativo-laborales completas de los 10 ex-alumnos entrevistados. 
los vayan renovando periódicamente o, al menos, que "les vayan llamando". Por ello es por lo que, cuando en páginas precedentes he presentado los rasgos del tipo de trabajos que les llevan a posponer los estudios universitarios, he utilizado la expresión "duración que virtualmente prometen" para designar justamente esa clase de situaciones, no atreviéndome a recurrir a la de "estabilidad", por cuanto ésta se la representan bajo la forma de contrato indefinido y/o del estatuto de funcionario, de manera que el mismo vocablo aparece en sus discursos tan sólo cuando hacen proyecciones a largo plazo ${ }^{8}$, tanto cuando se trata de quienes priorizan el trabajo, como de los que priman los estudios:

Yo no quiero seguir estudiando cuando termine educación social. Entonces, me gustaría empezar a trabajar como educadora o como integradora... A mí me gusta mucho el tema de menores. Vamos, yo es el tema en el que quiero hacer Prácticas de segundo y de tercero, y a ver si puedo seguir ahí, pero, vamos, que no descarto nada... Lo demás, pues, estupendo. Pero, vamos, eso es lo que me gustaría... ¡Vamos a ver! Yo el año pasado decía: «Pues, hago las oposiciones para centros de menores", pero... por lo que he oído de los centros de menores y por comentarios y por gente que está trabajando y tal, pues, que se te quitan mucho las ganas de trabajar en un centro de menores. Entonces, como de funcionaria en un Ayuntamiento yo no me veo, entonces, pues, no sé, me gustaría más en asociaciones o algo así... Eso también es como todo, ¿sabes? Es lo típico, que piensas: "Ahora que soy joven, pues, me apetecen cosas más dinámicas"; te apetece probar más cosas y tal. Quizá de cara al futuro, ya piensas en formar una familia, en más estabilidad y tal, pues, quizá sí digas «Pues, me voy a preparar unas oposiciones y tal.. (IS, promoción 1997-99, centro A)

Ahora bien, la estabilidad sólo la ven posible si se logra una plaza en la Administración Pública. Es más, ésta se percibe también como el lugar donde se puede conseguir una mayor duración de los empleos, amén de un salario más elevado, un trabajo social y/o educativo menos mercantilista, y unas relaciones laborales menos opresivas:

En las escuelas privadas se trabaja mucho más de cara a los padres, que son los clientes, que de los niños... En las privadas se nota mucho más que son los padres los que sueltan el dinero y, entonces, tienen que estar contentos los padres. Yo a M. (amiga que trabaja en una escuela privada) la veo muchas veces preocupada porque: "Se ha caído un niño. ¡Ya verás tú cuando venga el padre!, ique no sé cuál!’. Un día a mí me pasó que una niña le mordió en la espalda a otra niña, y sí que estaba preocupada... pero es que es inevitable: "Mira que ha pasado esto". Es decir, yo se lo explicaba (a los padres) y tal, pero, en cambio, el miedo que tienes en una empresa privada es mucho más. O sea, son unas relaciones de empresario-trabajador, no tú como responsable de tu trabajo, sino que la directora es

${ }^{8}$ Martín y Velarde (1996), en cambio, aseguran que no aparece nunca en los jóvenes investigados por ellos. 
la que manda... A mí, si es una escuela de la Comunidad de Madrid o, vamos, que sea pública - pero el sentido de lo público no porque a mí me parezca mejor lo público que lo privado, sino por las relaciones que se establecen a partir de eso, no me importaría trabajar en ello, pero tampoco me importaría trabajar como trabajadora social. (EI, promoción 1994-96, centro A)

Todo lo anterior hace inteligible que más de la mitad de los ex-alumnos hayan recurrido alguna vez a presentarse a un concurso-oposición, aun para optar a un contrato como eventuales, pero también su resistencia a abandonar los estudios cuando han conseguido éste $u$ otro cualquiera en la empresa privada o en el llamado tercer sector: para trabajar en intervención social y/o educativa, la Administración Pública "pide diplomaturas" - me decía un exalumno, cabe añadir "como mínimo" $-\mathrm{y}$, cuando no, sus poseedores juegan con ventaja:

El (curso) que hice de marginados sin hogar no me ha servido, porque entramos dos personas con una titulación inferior a diplomado, y ninguno de los dos trabajamos (en ello)... En cambio, el resto de la gente, que eran diplomados, educadores sociales o trabajadores sociales, sí empezaron a trabajar en el Ayuntamiento,... en diferentes programas o en diferentes lugares de intervención psico-social, ¿sabes? y el requisito imprescindible para hacer ese curso era FPII y, de hecho, yo creo que a mí me cogieron y a la otra gente, pues, para que Europa no dijera que no había nadie de FPII, (con ironía) simplemente que era muy difícil encontrarlos, pero que haber había, ¿sabes? Pero que es un curso que yo sé que no me va a servir en tanto en cuanto no sea diplomado como mínimo. (ASC, promoción 199395, centro B)

Así, desde mi punto de vista, es ese contraste entre el puesto de trabajo que satisface ahora y el que se anhela para el futuro el que se convierte, en el caso de los ex-alumnos que combinan estudios y trabajo, en una de las claves para comprender su "universitariomanía". Y es igualmente el que hace comprensible que, en vez de interrumpir los estudios, lleven unas vidas en extremo aceleradas y sin huecos entre sus múltiples ocupaciones: todo su tiempo parece estar ocupado por el binomio trabajo-universidad o, a veces, por el trinomio trabajo-universidad-formación complementaria. A modo ilustrativo, diré que, salvo uno (que fue entrevistado un domingo en su domicilio), me pidieron que les hiciera la entrevista en la Facultad donde cursan la carrera, perdiendo por ello una o dos clases, porque no encontraron en sus agendas otro momento para que pudiera hacérsela. La vocacionalización y/o el haber encontrado un trabajo que responda a las expectativas actuales, por consiguiente, no sólo no eliminan la "universitariomanía", sino que más bien la fomenta. 


\section{BIBLIOGRAFÍA CITADA}

ABDALA, E. 2001. Manual para la evaluación de impacto en programas de formación para jóvenes (versión borrador). CINTERFOR-OIT.

DensCOMBE, M. et alii. 1995. "Etnicidad y amistad. El contraste entre los estudios sociométricos y la observación directa en las aulas de primarian, en Peter Woods y Martyn Hammersley (comp.), Género, cultura y etnia en la escuela. Informes etnogrấficos. Barcelona: Paidós.

JOCILES, M. ISABEL. 2001. "Representaciones y modelos de formación en el profesorado de 'Servicios Socioculturales y a la Comunidad'. Politica y Sociedad.

Martín Serrano, Manuel. 1994. "Estudio y trabajo", en Historia de los cambios de mentalidad de los jóvenes entre 1960-1990. Madrid: INJUVE.

- y O. VELARDE. 1996. Informe Juventud España 96. Madrid: INJUVE.

Ministerio DE TRABajo y Asuntos Sociales. 2000. Nuevo Programa Nacional de Formación Profesional. Madrid: Ministerio de Trabajo y Asuntos Sociales. 